\title{
Radiation from a Point Charge Moving through a Circular Aperture in a Screen
}

\author{
P. M. VAN DEN BERG \\ Department of Electrical Engineering, Delft University of Technology, \\ Delft, The Netherlands
}

[Received 4 September 1974]

\begin{abstract}
This paper deals with the problem of diffraction radiation from an electric point charge that moves, at a constant speed, through a circular aperiure in a plane screen. The screen is assumed to be electrically perfectly conducting. The problem is reduced to the solution of an integral equation for an unknown field function in the aperture. Low-frequency solutions as well as high-frequency solutions are obtained. Results pertaining to the farfield behaviour of the radiated field are given. Finally, numerical results pertaining to the radiation loss of the charge are presented.
\end{abstract}

\section{Introduction}

THE energy lost by diffraction radiation when a charged particle passes a conducting structure is of considerable importance in accelerating structures. The explanation of the physical nature of this type of radiation is very simple. A point charge moving uniformly in a straight line in free space can be represented as a superposition of plane waves of different frequencies. All of the waves are evanescent waves decaying exponentially away from the trajectory of the particle. There is no radiation during this uniform motion of a charged particle. The presence of an ideally conducting body in the neighbourhood of the trajectory leads to diffraction of the evanescent waves. This diffracted field consists of waves either propagating or decaying exponentially from the obstacle. The propagating waves correspond to the diffraction radiation. The calculation of this field is rather difficult. Bolotovskii \& Voskresenskii $(1966,1968)$ and Hazeltine, Rosenbluth \& Sessler (1971) have reviewed the literature on this subject.

Hitherto, little consideration has been paid to the radiation of a charged particle passing through a circular aperture in a screen. Dnestrovskii \& Kostomarov (1959a) have considered the radiation of a modulated beam of charged particles passing through the circular aperture in a screen. They obtained an integral equation in which the diffracted field in the aperture and the incident field (field of the modulated beam of charged particles in free space) upon the screen occur. A numerical solution has been obtained in replacing the integral equation by a system of linear algebraic equations. The calculations were performed for values of $\alpha=k a$ between 0.5 and $4.0(k=$ $\omega / c_{0}=$ wave number of the radiation, $a=$ radius of the aperture). However, approximate solutions for small $\alpha$ and large $\alpha$ have not been presented, and in order to calculate the radiation loss of a single charged particle we need the behaviour for all values of $\alpha$. For a single charge with ultrarelativistic velocity, Dnestrovskii \& Kostomarov (1959b) and Hemp (1974) calculated the radiation loss. The approximation used by them is in fact an approximation for large $\alpha$ (in the case of ultrarelativistic velocity) and has been extrapolated to other values of $\alpha$. For a lower velocity of the charge no results are known. 
In the present paper, a new approach is provided with respect to the diffraction radiation from a point charge moving through a circular aperture in a plane screen. An integral equation is obtained, in which only aperture-field distributions occur. The integral equation is of the same form as the one occurring in the diffraction of a scalar plane wave by a soft disk. In the latter problem, techniques are used which transform the integral equation to one especially suitable either for handling low frequencies (Jones, 1956; Collins, 1961; Williams, 1962) or for handling high frequencies (Jones, 1965a; Newby, 1972; Williams, 1972). An extension of these techniques to our problem of the diffraction radiation from a point charge yields solutions for small $\alpha$ and large $\alpha$. From this, enough information can be obtained to calculate the radiation loss of the point charge. Finally, a number of numerical results pertaining to these radiation losses are presented for different velocities of the point charge.

\section{Formulation of the Problem}

The point charge $q$ under consideration moves with constant velocity $\mathbf{v}=v_{0} \mathbf{i}_{z}\left(v_{0}<\right.$ $c_{0}, c_{0}=$ velocity of light) along the axis of a system of circular cylindrical coordinates $(r, \phi, z)$ through the circular aperture of a screen. The screen at $z=0$ is assumed to be electrically perfectly conducting. The aperture occupies the region $z=0,0 \leqslant r \leqslant a$, all $\phi$. Since the geometrical configuration is independent of $\phi$, all field quantities are independent of $\phi$. To calculate the diffraction radiation from the charge, we start with a suitable representation of the field of the moving point charge in free space. This field $\mathbf{E}^{i}=\mathbf{E}^{i}(r, z, t), \mathbf{H}^{i}=\mathbf{H}^{i}(r, z, t)$ can be considered as the field incident upon the configuration. The first step is to represent the electric-and the magnetic-field vector as a Fourier integral:

$$
\begin{aligned}
\mathbf{E}^{i}(r, z, t) & =\frac{1}{\pi} \operatorname{Re}\left[\int_{0}^{\infty} \mathscr{E}^{i}(r, z ; \omega) \exp (i \omega t) d \omega\right], \\
\mathbf{H}^{i}(r, z, t) & =\frac{1}{\pi} \operatorname{Re}\left[\int_{0}^{\infty} \mathscr{H}^{i}(r, z ; \omega) \exp (i \omega t) d \omega\right] .
\end{aligned}
$$

From Maxwell's equation it follows that, since $\partial / \partial \phi=0$,

$$
\begin{aligned}
\mathscr{E}^{i} & =\frac{1}{i \omega \varepsilon_{0}}\left[\frac{\partial}{\partial z}\left(\frac{\partial \Pi^{i}}{\partial r}\right) \mathbf{i}_{r}-\frac{1}{r} \frac{\partial}{\partial r}\left(r \frac{\partial \Pi^{l}}{\partial r}\right) \mathbf{i}_{2}-\mathscr{F} \mathbf{i}_{z}\right], \\
\mathscr{H}^{i} & =-\frac{\partial \Pi^{i}}{\partial r} \mathbf{i}_{\phi},
\end{aligned}
$$

in which $\Pi^{i}=\Pi^{i}(r, z ; \omega)$ has to satisfy

$$
\nabla^{2} \Pi^{i}+k^{2} \Pi^{i}=-\mathscr{F} \quad\left(k=\omega\left(\varepsilon_{0} \mu_{0}\right)^{\frac{1}{2}}=\frac{\omega}{c_{0}}\right)
$$

and $\mathscr{F}=\mathscr{F}(r, z ; \omega)$ is the Fourier transform of the electric-current density in the $z$-direction:

$$
\mathscr{F}=\int_{-\infty}^{\infty} q v_{0} \delta\left(x, y, z-v_{0} t\right) \exp (-i \omega t) d t
$$




$$
=q \exp \left(-i \frac{\omega}{v_{0}} z\right) \delta(x, y) \text { with } r=\left(x^{2}+y^{2}\right)^{t} .
$$

The solution of (2.3) with (2.4) is obtained as

$$
\begin{aligned}
\Pi^{i} & =\frac{q}{2 \pi} \exp \left(-i \frac{\omega}{v_{0}} z\right) K_{0}\left\{\frac{\omega}{v_{0}}\left(1-\frac{v_{0}^{2}}{c_{0}^{2}}\right)^{\frac{1}{z}} r\right\} \\
& =\frac{q}{2 \pi} \exp \left(-i k \frac{c_{0}}{v_{0}} z\right) K_{0}\left\{k\left(\frac{c_{0}^{2}}{v_{0}^{2}}-1\right)^{\frac{1}{2}} r\right\} .
\end{aligned}
$$

We observe that the Fourier components of the field of the moving point charge are plane waves propagating in the $z$-direction with a phase velocity that equals the velocity of the charge. In the $r$-direction the amplitudes decay with increasing $r\left(v_{0}<c_{0}\right)$. This field cannot radiate in the $r$-direction.

The influence of the diffracting obstacle can now be explained by noting that these waves can be diffracted and thus can give rise to radiating plane waves representing the diffraction radiation of the moving charge. We now shall outline the diffraction problem. The total field is denoted by $\mathbf{E}=\mathbf{E}(r, z, t)$ and $\mathbf{H}=\mathbf{H}(r, z, t)$; then the diffracted radiated field is introduced as

$$
\mathbf{E}^{r} \equiv \mathbf{E}-\mathbf{E}^{i}, \quad \mathbf{H} \mathbf{r} \equiv \mathbf{H}-\mathbf{H}^{i} .
$$

This field, too, can be represented as a Fourier integral

$$
\begin{aligned}
& \mathbf{E}^{r}(r, z, t)=\frac{1}{\pi} \operatorname{Re}\left[\int_{0}^{\infty} \mathscr{E}^{r}(r, z ; \omega) \exp (i \omega t) d \omega\right], \\
& \mathbf{H}^{r}(r, z, t)=\frac{1}{\pi} \operatorname{Re}\left[\int_{0}^{\infty} \mathscr{H}^{r}(r, z ; \omega) \exp (i \omega t) d \omega\right] .
\end{aligned}
$$

Since the incident field possesses the property $H_{\phi}^{i} \neq 0$ and $E_{\phi}^{l}=0$ and the diffracting configuration is independent of $\phi$, it then follows from Maxwell's equations that the total field possesses the property $H_{\phi} \neq 0$ and $E_{\phi}=0$. The fundamental unknown is the Fourier component $\mathscr{H}_{\phi}=\mathscr{H}_{\phi}(r, z ; \omega)$ of the magnetic field vector in the $\phi$ direction. From the source-free Maxwell's equations it follows that

$$
\begin{aligned}
& \mathscr{E}^{r}=\frac{1}{i \omega \varepsilon_{0}}\left[\frac{\partial}{\partial z}\left(\frac{\partial \Pi^{r}}{\partial r}\right) \mathrm{i}_{r}-\frac{1}{r} \frac{\partial}{\partial r}\left(r \frac{\partial \Pi^{r}}{\partial r}\right) \mathbf{i}_{z}\right], \\
& \mathscr{H}^{r}=-\left(\frac{\partial \Pi^{r}}{\partial r}\right) \mathrm{i}_{\phi},
\end{aligned}
$$

in which $\Pi^{r}=\Pi^{r}(r, z ; \omega)$ satisfies

$$
\nabla^{2} \Pi^{r}+k^{2} \Pi^{r}=0 .
$$

The boundary conditions at the screen lead to

$$
\frac{\partial}{\partial z}\left(\mathscr{H}_{\phi}^{\prime}+\mathscr{H}_{\phi}^{r}\right)=0 \quad \text { at } z=0, a<r<\infty .
$$

Further, $\partial \mathscr{H}_{\phi} / \partial z$ is assumed to have a singularity of order $\left(a^{2}-r^{2}\right)^{-\frac{1}{t}}$ near the edge of the aperture in the plane $z=0$. At large distance from the aperture $\mathscr{H}_{\phi}^{r}$ is required to represent a disturbance travelling outwards.

In later work, we omit the symbol $\omega$ to denote that the quantities are dependent of $\omega$. 


\section{Integral Representations and Integral Equations}

A straightforward application of Green's theorem to the field functions, defined in Section 2, yields integral representations and an integral equation, in which field distributions upon the screen occur. We are then dealing with an infinite range of integration. We rather want an integral equation in which an aperture field occurs. Therefore, we make the following alternative approach. Application of Green's theorem to the domains $z<0$ and $z>0$, respectively, yields

with

$$
\begin{aligned}
\mathscr{H}_{\phi}^{r}\left(r_{0}, z_{0}\right)= & \mp \frac{\partial}{\partial r_{0}} \int_{0}^{a} r\left[\frac{\partial \Pi^{r}(r, z)}{\partial z}\right]_{z=0} G\left(r_{0}, z_{0} ; r, 0\right) d r \\
& \pm \frac{\partial}{\partial r_{0}} \int_{a}^{\infty} r\left[\frac{\partial \Pi^{i}(r, z)}{\partial z}\right]_{z=0} G\left(r_{0}, z_{0} ; r, 0\right) d r, \quad z_{0} \lessgtr 0,
\end{aligned}
$$

$$
G\left(r_{0}, \dot{z}_{0} ; r, z\right)=\int_{0}^{2 \pi}\left[\frac{\exp \left(-i k\left|\mathbf{r}_{0}-\mathbf{r}\right|\right)}{4 \pi\left|\mathbf{r}_{0}-\mathbf{r}\right|}+\frac{\exp \left(-i k\left|\mathbf{r}_{0}^{\prime}-\mathbf{r}\right|\right)}{4 \pi\left|\mathbf{r}_{0}^{\prime}-\mathbf{r}\right|}\right] d\left(\phi_{0}-\phi\right),
$$

in which $\mathbf{r}_{0}=\left(r_{0}, \phi_{0}, z_{0}\right), \mathbf{r}_{0}^{\prime}=\left(r_{0}, \phi_{0},-z_{0}\right)$ and $\mathbf{r}=(r, \phi, z)$. In the same manner, application of Green's theorem to the domains $z<0$ and $z>0$ outside the region $r<\varepsilon(\varepsilon \rightarrow 0)$ yields

$$
\begin{aligned}
\mathscr{H}_{\phi}^{i}\left(r_{0}, \mp z_{0}\right) & =\lim _{\varepsilon \rightarrow 0}\left\{\mp \frac{\partial}{\partial r_{0}} \int_{e}^{\infty} r\left[\frac{\partial \Pi^{i}(r, \mp z)}{\partial z}\right]_{z=0} G\left(r_{0}, z_{0} ; r, 0\right) d r\right\}, \\
& =\frac{\partial}{\partial r_{0}} \int_{0}^{\infty} r\left[\frac{\partial \Pi^{i}(r, z)}{\partial z}\right]_{z=0} G\left(r_{0}, z_{0} ; r, 0\right) d r, \quad z_{0} \lessgtr 0, r_{0} \neq 0 .
\end{aligned}
$$

Combining the results of (3.1) and (3.3) in such a way that the integrals over the screen cancel, and then requiring that in the aperture $\mathscr{H}_{\phi}^{\prime}$ has to be continuous, we obtain the integral equation

$\mathscr{H}_{\phi}^{i}\left(r_{0}, 0\right)=\frac{\partial}{\partial r_{0}} \int_{0}^{a} r\left[\frac{\partial \Pi^{i}(r, z)}{\partial z}+\frac{\partial \Pi^{r}(r, z)}{\partial z}\right]_{z=0} G\left(r_{0}, 0 ; r, 0\right) d r, \quad 0<r_{0}<a$.

Integrating with respect to $r_{0}$, and introducing the dimensionless variables $\rho_{0}=r_{0} / a$, $\rho=r / a$ and $\alpha=k a$, we obtain

with

$$
\int_{0}^{1} \rho f(\rho) K\left(\rho_{0}, \rho\right) d \rho=C+\Pi^{i}\left(a \rho_{0}, 0\right), \quad 0<\rho_{0}<1,
$$

and

$$
\rho f(\rho)=i \alpha \frac{c_{0}}{v_{0}} \rho \Pi^{i}(a \rho, 0)-\rho\left[a \frac{\partial \Pi^{\prime}(a \rho, z)}{\partial z}\right]_{z=0},
$$

$$
K\left(\rho_{0}, \rho\right)=\int_{0}^{2 \pi} \frac{\exp \left\{-i \alpha\left(\rho_{0}^{2}-2 \rho_{0} \rho \cos \phi+\rho^{2}\right)^{\frac{1}{2}}\right\}}{2 \pi\left(\rho_{0}^{2}-2 \rho_{0} \rho \cos \phi+\rho^{2}\right)^{\frac{1}{2}}} d \phi .
$$

$C$ is an arbitrary constant and will be determined by the condition that $d f / d \rho$ is $O\left(\left(1-\rho^{2}\right)^{-\frac{1}{2}}\right)$ near the edge of the aperture $(\rho=1)$. We observe, further, that $\Pi^{i}(a \rho, 0)$ can be written as

$$
\Pi^{i}(a \rho, 0)=\frac{q}{2 \pi} K_{0}(\alpha \Gamma \rho), \quad \text { with } \quad \Gamma=\left(\frac{c_{0}^{2}}{v_{0}^{2}}-1\right)^{\frac{1}{2}} .
$$


In Section 5 we shall discuss the solution of integral equation (3.5) for small $\alpha$ and in Section 6 we shall discuss the solution for large $\alpha$.

\section{Far-field Behaviour-Radiation Loss of the Point Charge}

In order to calculate the radiation loss, we first consider the far-field behaviour of the radiation field. Since $\Pi^{i}$ has the exponentially decaying behaviour of a $K_{0}$-function in the $r$-direction, only finite values of $r$ contribute significantly to the second integral in (3.1), when $k \Gamma \neq 0$. For values of $r \gg 1 / k \Gamma$ the contribution to the integration can then be neglected. Hence, we can now write

$$
\begin{gathered}
\mathscr{H}_{\phi}^{r}\left(r_{0}, \mp z_{0}\right)= \pm A(\theta) \frac{\exp (-i k R)}{R}+O\left(\frac{1}{R^{2}}\right), \\
r_{0}=R \sin \theta \text { and } z_{0}=R \cos \theta \text { as } R \rightarrow \infty, 0 \leqslant \theta<\frac{1}{2} \pi,
\end{gathered}
$$

in which, using the relation $\int_{0}^{2 \pi} \exp (i k r \sin \theta \cos \phi) d \phi=2 \pi J_{0}(k r \sin \theta)$,

$$
\begin{aligned}
A(\theta) & \simeq i k \sin \theta \int_{0}^{a} r\left[\frac{\partial \Pi^{r}(r, z)}{\partial z}\right]_{z=0} J_{0}(k r \sin \theta) d r \\
& -i k \sin \theta \int_{a}^{r_{m}} r\left[\frac{\partial \Pi^{i}(r, z)}{\partial z}\right]_{z=0} J_{0}(k r \sin \theta) d r, \quad \frac{1}{k \Gamma} \ll r_{m} \ll R .
\end{aligned}
$$

Introduction of dimensionless variables leads to

$$
\begin{aligned}
A(\theta) & \simeq i \alpha \sin \theta \int_{0}^{1} \rho\left[a \frac{\partial \Pi^{r}(a \rho, z)}{\partial z}\right]_{z=0} J_{0}(\alpha \rho \sin \theta) d \rho \\
& -\frac{q}{2 \pi} \alpha^{2} \frac{c_{0}}{v_{0}} \sin \theta \int_{1}^{\rho_{m}} \rho K_{0}(\alpha \Gamma \rho) J_{0}(\alpha \rho \sin \theta) d \rho, \quad \frac{1}{\alpha \Gamma} \ll \rho_{m} .
\end{aligned}
$$

In Sections 5 and 6 , this expression will be considered further. Let us now calculate the radiated energy. This energy $Q$ is twice the total energy radiated along the hemisphere radius $R, 0 \leqslant \theta<\frac{1}{2} \pi$, all $\phi$, across $S$, the curved surface of the hemisphere,

$$
\begin{aligned}
Q & =2 \iint_{S} \int_{-\infty}^{\infty}\left(\mathrm{E}^{r} \times \mathbf{H}^{r}\right) \cdot \mathbf{i}_{R} d t d S \\
& =\int_{0}^{\infty} P(\omega) d \omega,
\end{aligned}
$$

in which, using (2.7),

$$
P(\omega)=\frac{2}{\pi} \operatorname{Re}\left[\iint_{S}\left(\mathscr{E}^{r} \times \mathscr{H}^{r^{*}}\right) \cdot \mathbf{i}_{R} d S\right],
$$

(asterisk denotes complex conjugate).

The quantity $P(\omega) d \omega$ relates to the radiation loss of the moving charge, caused by radiation with frequencies between $\omega$ and $\omega+d \omega$. From Maxwell's equations in spherical polar coordinates $(R, \theta, \phi)$, it then follows $\left(\partial / \partial \phi=0, \mathscr{H}_{R}^{r}=0, \mathscr{H}_{\theta}^{r}=0\right)$,

$$
\mathscr{E} r=\frac{1}{i \omega \varepsilon_{0}}\left[\frac{1}{R \sin \theta} \frac{\partial}{\partial \theta}\left(\sin \theta \mathscr{H}_{\phi}^{r}\right) \mathbf{i}_{R}-\frac{1 \partial}{R \partial R}\left(R \mathscr{H}_{\phi}^{r}\right) \mathbf{i}_{\theta}\right]
$$


hence, with (4.1),

$$
\begin{aligned}
P(\omega) & =4 \operatorname{Re}\left[\int_{0}^{\frac{1}{2} \pi} \mathscr{E}_{\theta} \mathscr{H}_{\phi}^{r *} R^{2} \sin \theta d \theta\right] \\
& =4\left(\frac{\mu_{0}}{\varepsilon_{0}}\right)^{\frac{1}{2}} \int_{0}^{\frac{1}{2} \pi}|A(\theta)|^{2} \sin \theta d \theta,
\end{aligned}
$$

in which $\left(\mu_{0} / \varepsilon_{0}\right)^{t} \simeq 120 \pi$ is the wave impedance of free space. We observe that the radiation loss can be calculated once $A(\theta)$ has been determined from (4.3), and hence as soon as $[\partial \Pi r(r, z) / \partial z]_{z=0}$ has been determined.

\section{Solution for Small $\alpha$}

The solution of an integral equation like (3.5) with the kernel (3.6) can be transformed into a solution of a Fredholm integral equation of the second kind, which is very suitable for small $\alpha$ (Jones, 1956; Collins, 1961; Williams, 1962). Along the same line, we now find that (3.5) with (3.6) can be transformed into

$$
\begin{aligned}
W(v)=\frac{d}{d v} \int_{0}^{v} w\left\{C+\Pi^{i}(a w, 0)\right\} & \frac{\cosh \left\{\alpha\left(v^{2}-w^{2}\right)^{\frac{1}{t}}\right\}}{\left(v^{2}-w^{2}\right)^{\frac{1}{2}}} d w- \\
& \int_{0}^{1} W(w) L(v, w) d w, \quad 0<v<1,
\end{aligned}
$$

where

$$
L(v, w)=-\frac{i}{\pi}\left[\frac{\sinh \{\alpha(v+w)\}}{v+w}+\frac{\sinh \{\alpha(v-w)\}}{v-w}\right],
$$

and

$$
W(v)=\int_{v}^{1} \rho f(\rho) \frac{\cos \left\{\alpha\left(\rho^{2}-v^{2}\right)^{\frac{1}{2}}\right\}}{\left(\rho^{2}-v^{2}\right)^{\frac{1}{2}}} d \rho .
$$

Equation (5.3) may be inverted to give (Jones, 1956)

$$
f(\rho)=-\frac{2}{\pi} \frac{1}{\rho} \frac{d}{d \rho} \int_{\rho}^{1} v W(v) \frac{\cosh \left\{\alpha\left(v^{2}-\rho^{2}\right)^{\frac{1}{2}}\right\}}{\left(v^{2}-\rho^{2}\right)^{\frac{1}{2}}} d v,
$$

and hence in order that $d f / d \rho$ is $O\left(\left(1-\rho^{2}\right)^{-1}\right)$ near the edge of the aperture $(\rho=1)$, it follows from an integration by parts that we have to require that $W(1)=0$. This condition determines the arbitrary constant $C$ and equation (5.1) thus becomes

$$
\begin{array}{r}
W(v)=\frac{\cosh (\alpha v)}{\cosh \alpha} B(1)-B(v)+\int_{0}^{1} W(w)\left\{\frac{\cosh (\alpha v)}{\cosh \alpha} L(1, w)-L(v, w)\right\} d w, \\
0<v<1,
\end{array}
$$

in which, using (3.7),

$$
B(v)=-\frac{q}{2 \pi} \frac{d}{d v} \int_{0}^{v} w K_{0}(\alpha \Gamma w) \frac{\cosh \left\{\alpha\left(v^{2}-w^{2}\right)^{\frac{1}{2}}\right\}}{\left(v^{2}-w^{2}\right)^{\frac{1}{2}}} d w .
$$

The practical values of interest are the values of $c_{0} / v_{0}$ not too large, say $1<c_{0} / v_{0}<2$. From the Appendix, it then follows that the first terms in an asymptotic development of $B(v)$ for small $\alpha$ are 


$$
\begin{gathered}
B(v)=\frac{q}{2 \pi}\left[\gamma+\ln (\alpha \Gamma v)-\frac{1}{4} \alpha^{2} \Gamma^{2} v^{2}+\frac{1}{2} \alpha^{2}\left(\Gamma^{2}+1\right) v^{2}\{-1+\gamma+\ln (\alpha \Gamma v)\}+o\left(\alpha^{3}\right)\right] \\
\gamma=0.577216 \ldots, \text { Euler's constant, }
\end{gathered}
$$

and hence

$$
\begin{aligned}
\frac{\cosh (\alpha v)}{\cosh \alpha} B(1)-B(v)= & B(1)-B(v)-\frac{\alpha^{2}}{2}\left(1-v^{2}\right) B(1)+O\left(\alpha^{4}\right) \\
& =\frac{q}{2 \pi}\left[-\ln v+\frac{1}{2} \alpha^{2} \Gamma^{2}\left(1-v^{2}\right) \ln (\alpha \Gamma)-\frac{1}{2} \alpha^{2}\left(\Gamma^{2}+1\right) v^{2} \ln v+\right. \\
& \left.\frac{1}{2} \alpha^{2}\left(1-v^{2}\right)\left\{\Gamma^{2}\left(-\frac{3}{2}+\gamma\right)-1\right\}+o\left(\alpha^{3}\right)\right]
\end{aligned}
$$

The asymptotic development of the kernel of integral equation (5.5) for small $\alpha$ becomes

$$
\frac{\cosh (\alpha v)}{\cosh \alpha} L(1, w)-L(v, w)=\frac{2 i}{3 \pi} \alpha^{3}\left(1-v^{2}\right)+O\left(\alpha^{5}\right) .
$$

The first few terms of the iterative solution of equation (5.5) are then obtained as

$$
\begin{aligned}
& W(v)=\frac{q}{2 \pi}\left[-\ln v+\frac{1}{2} \alpha^{2} \Gamma^{2}\left(1-v^{2}\right) \ln (\alpha \Gamma)-\frac{1}{2} \alpha^{2}\left(\Gamma^{2}+1\right) v^{2} \ln v+\right. \\
& \left.\frac{1}{2} \alpha^{2}\left(1-v^{2}\right)\left\{\Gamma^{2}\left(-\frac{3}{2}+\gamma\right)-1\right\}+\frac{2 i}{3 \pi} \alpha^{3}\left(1-v^{2}\right)+o\left(\alpha^{3}\right)\right] .
\end{aligned}
$$

Now, in order to obtain the amplitude $A(\theta)$ of the radiation field, (4.3) can be rewritten as

$$
\begin{aligned}
A(\theta) \simeq-\frac{q}{2 \pi} \alpha^{2} \frac{c_{0}}{v_{0}} & \sin \theta \int_{0}^{\rho_{m}} \rho K_{0}(\alpha \Gamma \rho) J_{0}(\alpha \rho \sin \theta) d \rho- \\
& \quad i \alpha \sin \theta \int_{0}^{1} \rho f(\rho) J_{0}(\alpha \rho \sin \theta) d \rho, \quad \frac{1}{\alpha \Gamma} \ll \rho_{m} .
\end{aligned}
$$

The first integral can be integrated analytically, using a result due to Lommel (Watson, 1966 , p. 134). The second integral can be rewritten, using

$$
J_{0}(\alpha \rho \sin \theta)=\frac{2}{\pi} \int_{0}^{\rho} \cosh (\alpha v \cos \theta) \frac{\cos \left\{\alpha\left(\rho^{2}-v^{2}\right)^{\frac{1}{2}}\right\}}{\left(\rho^{2}-v^{2}\right)^{\frac{1}{2}}} d v
$$

following from Sonine's second finite integral (Watson, 1966, p. 376). We obtain with (5.3) after a slight manipulation

$$
\begin{aligned}
A(\theta) \simeq & -\frac{q}{2 \pi} \frac{c_{0}}{v_{0}} \frac{\sin \theta}{\Gamma^{2}+\sin ^{2} \theta} \times \\
& {\left[\alpha \rho \sin \theta J_{1}(\alpha \rho \sin \theta) K_{0}(\alpha \Gamma \rho)-\alpha \Gamma \rho K_{1}(\alpha \Gamma \rho) J_{0}(\alpha \rho \sin \theta)\right]_{p=0}^{\rho=\rho_{m}}-} \\
& \frac{2 i}{\pi} \alpha \sin \theta \int_{0}^{1} W(v) \cosh (\alpha v \cos \theta) d v, \quad \frac{1}{\alpha \Gamma} \ll \rho_{m} .
\end{aligned}
$$

The expression between the brackets gives a zero contribution at $\rho=\rho_{m} \gg 1 / \alpha \Gamma$, approximately, and gives the contribution -1 at $\rho=0$. With the result of (5.10), we then obtain the first few terms of an asymptotic development for small $\alpha$, 


$$
\begin{gathered}
A(\theta)=\frac{q}{2 \pi}\left[-\frac{c_{0}}{v_{0}} \frac{\sin \theta}{\Gamma^{2}+\sin ^{2} \theta}-\frac{2 i}{\pi} \alpha \sin \theta-\frac{2 i}{3 \pi} \alpha^{3} \Gamma^{2} \ln (\alpha \Gamma) \sin \theta+\right. \\
\left.\frac{i}{9 \pi} \alpha^{3}\left\{\Gamma^{2}(8-6 \gamma)+5-\cos ^{2} \theta\right\} \sin \theta+\frac{8}{9 \pi^{2}} \alpha^{4} \sin \theta+o\left(\alpha^{4}\right)\right], \quad \alpha \Gamma \neq 0 .
\end{gathered}
$$

This is the required expression for the far-amplitude of the radiation field, in case $\alpha$ is small, $\Gamma$ not too large, but $\alpha \Gamma \neq 0$. From (4.7), we find

$$
\begin{array}{r}
P(\omega)=4\left(\frac{\mu_{0}}{\varepsilon_{0}}\right)^{\frac{1}{2}}\left(\frac{q}{2 \pi}\right)^{2} \int_{0}^{\frac{1}{2} \pi}\left[\frac{c_{0}^{2}}{v_{0}^{2}} \frac{1}{\left(\Gamma^{2}+\sin ^{2} \theta\right)^{2}}+\frac{4}{\pi^{2}} \alpha^{2}+\frac{8}{3 \pi^{2}} \alpha^{4} \Gamma^{2} \ln (\alpha \Gamma)-\right. \\
\left.\frac{4}{9 \pi^{2}} \alpha^{4}\left\{\Gamma^{2}(8-6 \gamma)+5-\cos ^{2} \theta+\frac{c_{0}}{v_{0}} \frac{4}{\Gamma^{2}+\sin ^{2} \theta}\right\}+\cdots\right] \sin _{\alpha \Gamma d \theta}+\cdots 0 \\
\alpha \Gamma \neq 0
\end{array}
$$

with

$$
\alpha=\omega \frac{a}{c_{0}} .
$$

The integrals occurring in (5.14) can be carried out analytically. We obtain

$$
\begin{aligned}
P(\omega)= & \left(\frac{\mu_{0}}{\varepsilon_{0}}\right)^{ \pm}\left(\frac{q}{\pi}\right)^{2}\left[\frac{1}{4}\left(\frac{c_{0}}{v_{0}}+\frac{v_{0}}{c_{0}}\right) \ln \frac{\left(c_{0} / v_{0}\right)+1}{\left(c_{0} / v_{0}\right)-1}-\frac{1}{2}+\right. \\
& \frac{8}{3 \pi^{2}} \alpha^{2}+\frac{16}{9 \pi^{2}} \alpha^{4} \Gamma^{2} \ln (\alpha \Gamma)-\frac{8}{27 \pi^{2}} \alpha^{4}\left\{\Gamma^{2}(8-6 \gamma)+5\right\}+ \\
& \left.\frac{8}{135 \pi^{2}} \alpha^{4}-\frac{16}{9 \pi^{2}} \alpha^{4}\left\{\frac{c_{0}}{v_{0}}-\frac{1}{2} \Gamma^{2} \ln \frac{\left(c_{0} / v_{0}\right)+1}{\left(c_{0} / v_{0}\right)-1}\right\}+\cdots\right], \alpha \Gamma \neq 0 .
\end{aligned}
$$

The first two terms in (5.15) are $\alpha$-independent and coincide with the case of transition radiation for a charged particle passing through an ideal screen. The other terms represent corrections to this point of view. Some numerical results will be presented in Section 7.

\section{Solution for Large $\alpha$}

Integral equation (3.5) with the kernel (3.6) can be transformed into an integral equation suitable for large $\alpha$ (Jones, 1965a)

$$
\int_{0}^{1} w f(w)\left[\frac{\exp \{-i \alpha(w+v)\}}{w+v}+\frac{\exp \{-i \alpha(w-v)\}}{w-v}\right] d w=F_{0}(v), \quad 0<v<1,
$$

where

$$
F_{0}(v)=2 \frac{d}{d v} \int_{0}^{v} \frac{\cos \left\{\alpha\left(v^{2}-w^{2}\right)^{\frac{1}{2}}\right\}}{\left(v^{2}-w^{2}\right)^{\frac{1}{2}}} w \frac{d}{d w} \int_{0}^{w} \rho\left\{C+\Pi^{i}(a \rho, 0)\right\} \frac{\cosh \left\{\alpha\left(w^{2}-\rho^{2}\right)^{\frac{1}{2}}\right\}}{\left(w^{2}-\rho^{2}\right)^{\frac{1}{2}}} d \rho d w .
$$

In (6.1) a Cauchy principal value is understood. In later work, a principal value is always implied when an integrand contains a simple pole on the interval of integration. By letting

$$
F(v)=\int_{0}^{1} w f(w) \frac{\exp \{-i \alpha(w-v)\}}{w-v} d w
$$


Jones (1965a) converted the integral equation (6.1) into a Fredholm integral equation of the second kind:

$$
F(v)=F_{0}(v)-\frac{1}{\pi}\left(\frac{v}{1+v}\right)^{\frac{1}{2}} \int_{0}^{1}\left(\frac{1-w}{w}\right)^{\frac{1}{2}} F(w) \frac{\exp \{-i \alpha(w+v)\}}{w+v} d w .
$$

Inversion of (6.3) and application of the condition that $w f(w)$ should be zero at the origin shows that

$$
w f(w)=-\frac{1}{\pi^{2}}\left(\frac{w}{1-w}\right)^{\frac{1}{2}} \int_{0}^{1}\left(\frac{1-v}{v}\right)^{\frac{1}{2}} F(v) \frac{\exp \{-i \alpha(v-w)\}}{v-w} d v .
$$

Jones (1965a) has also devised a special iterative procedure to obtain a solution of (6.4) for large $\alpha$. To obtain the first iteration is rather difficult; see also Thomas (1968) and Newby (1972). Williams (1972) has shown that the first iteration can be obtained in a more direct way, related to trivial boundary value problems, which arise in the absence of the diffracting screen. Therefore in our case, we consider the trivial boundary value problem in relation to the field

$$
u_{0}(r, z)=C \exp (-i k z)+\Pi^{i}(r, z) \text {, }
$$

in which $\Pi^{i}(r, z)$ is given by (2.5). Using Green's theorem in the domain $z>0, r \neq 0$, we have

$$
u_{0}\left(r_{0}, 0\right)=-\int_{0}^{\infty} r\left[\frac{\partial u_{0}(r, z)}{\partial z}\right]_{z=0} \int_{0}^{2 \pi} \frac{\exp \left\{-i k\left(r_{0}^{2}-2 r_{0} r \cos \phi+r^{2}\right)^{\frac{1}{2}}\right\}}{2 \pi\left(r_{0}^{2}-2 r_{0} r \cos \phi+r^{2}\right)^{\frac{1}{2}}} d \phi d r
$$

which, by introducing dimensionless variables, can be written as

$$
C+\Pi^{i}\left(a \rho_{0}, 0\right)=\int_{0}^{\infty} \rho h(\rho) K\left(\rho_{0}, \rho\right) d \rho,
$$

with $h(\rho)=i x C+i \alpha\left(c_{0} / v_{0}\right) \Pi^{i}(a \rho, 0)$, and with $K\left(\rho_{0}, \rho\right)$ is given by (3.6). In the same manner as equation (3.5) can be transformed into equation (6.1), equation (6.7) can be transformed into equation

$$
F_{0}(v)=\int_{0}^{\infty} w h(w)\left[\frac{\exp \{-i \alpha(w+v)\}}{w+v}+\frac{\exp \{-i \alpha(w-v)\}}{w-v}\right] d w, \quad 0<v<\infty .
$$

This expression of $F_{0}(v)$ is now very useful in the first step of Jones's iteration scheme to solve equation (6.5). With this iteration scheme, the solution of (6.5) becomes

where

$$
F(v)=\sum_{n=0}^{\infty} \int_{0}^{\infty} H_{n}(w) \frac{\exp \{-i \alpha(w-v)\}}{w-v} d w
$$

$$
H_{n}(w) \begin{cases}=w h(w)=i \alpha w\left[C+\frac{c_{0}}{v_{0}} \Pi^{i}(a w, 0)\right] & (n=0), \\ =\frac{i \alpha}{4 \pi} \int_{0}^{\infty}\left[\frac{v}{1+v}\right]^{t} h_{n}(v) \exp (-i \alpha v) M(v, w) d v & (n \geqslant 1),\end{cases}
$$

with .

$$
h_{n}(v)=\int_{1}^{\infty}\left(\frac{w-1}{w}\right)^{\frac{1}{2}} \frac{\exp (-i \alpha w)}{w+v} H_{n-1}(w) d w \quad(n \geqslant 1)
$$


and

$$
M(v, w)=\frac{4 w}{v^{2}-w^{2}}\left[v H_{1}^{(2)}(\alpha v) J_{0}(\alpha w)-w H_{0}^{(2)}(\alpha v) J_{1}(\alpha w)\right] .
$$

From (6.5) and the equations above, it then follows that

$$
w f(w)=\sum_{n=0}^{\infty}\left\{H_{n}(w)+\frac{1}{\pi}\left(\frac{w}{1-w}\right)^{\frac{1}{2}} \exp (i \alpha w) h_{n+1}(-w)\right\} .
$$

The constant $C$ can now be determined by requiring that $d f / d w$ is $O\left((1-w)^{-\frac{1}{3}}\right)$ at $w=1$. Hence, see also Thomas (1968) and Jones (1965b),

$$
\sum_{n=0}^{\infty} \exp (i \alpha) h_{n+1}(-1)=\sum_{n=0}^{\infty} \int_{1}^{\infty} \frac{\exp \{-i \alpha(w-1)\}}{w^{\frac{1}{2}}(w-1)^{\frac{1}{2}}} H_{n}(w) d w=0 .
$$

This condition determines the constant $C$.

Let us now return to the far-field amplitude $A(\theta)$. From (4.3) and (6.13), we obtain

$$
\begin{aligned}
A(\theta) \simeq & -\frac{q}{2 \pi} \alpha^{2} \frac{c_{0}}{v_{0}} \sin \theta \int_{1}^{\rho_{m}} \rho K_{0}(\alpha \Gamma \rho) J_{0}(\alpha \rho \sin \theta) d \rho+ \\
& \alpha^{2} C \sin \theta \int_{0}^{1} \rho J_{0}(\alpha \rho \sin \theta) d \rho- \\
& \frac{i}{\pi} \alpha \sin \theta \int_{0}^{1}\left(\frac{\rho}{1-\rho}\right)^{\frac{1}{2}} \exp (i \alpha \rho) h_{1}(-\rho) J_{0}(\alpha \rho \sin \theta) d \rho- \\
& i \alpha \sin \theta \sum_{n=1}^{\infty}\left[\int_{0}^{1} H_{n}(\rho) J_{0}(\alpha \rho \sin \theta) d \rho+\right. \\
& \left.\frac{1}{\pi} \int_{0}^{1}\left(\frac{\rho}{1-\rho}\right)^{\frac{1}{2}} \exp (i \alpha \rho) h_{n+1}(-\rho) J_{0}(\alpha \rho \sin \theta) d \rho\right], \frac{1}{\alpha \Gamma} \leqslant \rho_{m} .
\end{aligned}
$$

The first two integrals can be calculated analytically (Watson, 1966, pp. 132-134) and the other integrals can be rewritten as integrals from 1 to infinity (Newby, 1972), and hence,

$$
\begin{aligned}
A(\theta)=\frac{q}{2 \pi} & \frac{c_{0}}{v_{0}} \sin \theta \frac{\alpha \sin \theta J_{1}(\alpha \sin \theta) K_{0}(\alpha \Gamma)-\alpha \Gamma K_{1}(\alpha \Gamma) J_{0}(\alpha \sin \theta)}{\Gamma^{2}+\sin ^{2} \theta}+\alpha C J_{1}(\alpha \sin \theta)+ \\
& \frac{i}{\pi} \alpha \sin \theta \int_{1}^{\infty}\left(\frac{w}{1-w}\right)^{\frac{1}{2}} \exp (i \alpha w) h_{1}(-w) J_{0}(\alpha w \sin \theta) d w+ \\
& i \alpha \sin \theta \sum_{n=1}^{\infty}\left[\int_{1}^{\infty} H_{n}(w) J_{0}(\alpha w \sin \theta) d w+\right. \\
& \left.\frac{1}{\pi} \int_{1}^{\infty}\left(\frac{w}{1-w}\right)^{\frac{1}{2}} \exp (i \alpha w) h_{n+1}(-w) J_{0}(\alpha w \sin \theta) d w\right], \quad \alpha \Gamma \neq 0 .
\end{aligned}
$$

It is apparent that the far field of the radiation field is determined entirely from conditions at the edge of the aperture.

Let us now consider what happens when $\alpha$ is large. It is then possible to obtain an explicit expression for the field distribution (6.13) in the aperture and the far field amplitude (6.16) correct to any order desired. All that is required is to calculate integrals asymptotically to as many terms as are necessary. However, firstly we are 
interested in the leading terms. The first two terms of the asymptotic development of the field $w f(w)$ are given by

$w f(w) \simeq H_{0}(w)+\frac{1}{\pi}\left(\frac{w}{1-w}\right)^{\frac{1}{2}} \exp \left\{i \alpha(w-1)-i \frac{1}{4} \pi\right\} \int_{01}^{\infty} \frac{v^{\frac{1}{2}} \exp (-\alpha v)}{1-w-i v} H_{0}(1-i v) d v$,

$v$ not being replaced by zero in the denominator since $1-w$ vanishes at the edge of the aperture, and $v$ not directly being replaced by zero in $H_{0}(1-i v)$ because of the exponential behaviour of the modified Hankel function occurring in $H_{0}(w)$; hence we write $H_{0}(w)$ as

$$
H_{0}(w)=i w \alpha\left[C+\frac{q}{2 \pi} \frac{c_{0}}{v_{0}} \exp (-\alpha \Gamma w) S(\alpha \Gamma w)\right] \text { with } S(x)=\exp (x) K_{0}(x) .
$$

The slowly varying function $S(x)$ has been tabulated (Watson, 1966). Now $S(1-i v)$ may be replaced by $S(1)$ in (6.17). Using the relationship

$$
\begin{gathered}
\int_{0}^{\infty} \frac{w^{v} \exp (-\mu w)}{v-i w} d w=v ! i \exp (i \mu v) \int_{\mu}^{\infty} \frac{\exp (-i v x)}{x^{v+1}} d x \\
v>-1, \quad \operatorname{Re}(\mu)>0 \text { and } v>0
\end{gathered}
$$

we obtain

$$
\begin{array}{r}
w f(w) \simeq H_{0}(w)+\frac{\alpha}{2 \pi^{\frac{1}{3}}}\left(\frac{w}{1-w}\right)^{\frac{1}{2}} \exp \left(\frac{1}{4} \pi i\right)\left[C \int_{\alpha}^{\infty} \frac{\exp \{-i(1-w) x\}}{x^{\frac{3}{2}}} d x+\right. \\
\left.\frac{q}{2 \pi} \frac{c_{0}}{v_{0}} \exp (-\alpha \Gamma w) S(\alpha \Gamma) \int_{\alpha(1-i \Gamma)}^{\infty} \frac{\exp \{-i(1-w) x\}}{x^{\frac{3}{2}}} d x\right] .
\end{array}
$$

In the same approximation, the constant $C$ can be determined from (6.14),

$$
\exp (i \alpha) h_{1}(-1) \simeq \exp (i+\pi) \int_{0}^{\infty} \frac{\exp (-\alpha v)}{v^{\ddagger}} H_{0}(1-i v) d v \simeq 0 .
$$

Using (6.18) we then obtain

$$
C \simeq-\frac{q}{2 \pi} \frac{c_{0}}{v_{0}} \frac{\exp (-\alpha \Gamma) S(\alpha \Gamma)}{(1-i \Gamma)^{\frac{1}{2}}}=-\frac{q}{2 \pi} \frac{c_{0}}{v_{0}} \frac{K_{0}(\alpha \Gamma)}{(1-i \Gamma)^{\frac{1}{2}}},
$$

and this expression has to be used in (6.19). It can be shown that (6.19) with (6.21) gives the same distribution near the edge as if it were assumed that such a region behaved as a semi-infinite plane with edge along the tangent, placed in the incident field. The lower order terms omitted in (6.13) and (6.14) represent corrections to this point of view.

To the same degree of approximation as above, the function $h_{1}(-1) \simeq 0$; hence, only the two first terms of (6.16) remain and

$$
\begin{aligned}
& A(\theta) \simeq \frac{q}{2 \pi} \frac{c_{0}}{v_{0}} \alpha\left[\sin \theta \frac{\sin \theta J_{1}(\alpha \sin \theta) K_{0}(\alpha \Gamma)-\Gamma K_{1}(\alpha \Gamma) J_{0}(\alpha \sin \theta)}{\Gamma^{2}+\sin ^{2} \theta}-\right. \\
& \left.\frac{J_{0}(\alpha \sin \theta) K_{0}(\alpha \Gamma)}{(1-i \Gamma)^{\frac{t}{2}}}\right], \quad \alpha \Gamma \neq 0 .
\end{aligned}
$$

The calculation of the radiation loss at high frequencies from (4.7) and (6.22) will be carried out numerically. Numerical results will be presented in Section 7 . We 
remark that in case of ultrarelativistic velocity of the charge $\left(c_{0} / v_{0} \rightarrow 1\right.$ and $\left.\Gamma \rightarrow 0\right)$, expression (6.22) can be rewritten as

$$
A(\theta) \simeq-\frac{q}{2 \pi} \frac{c_{0}}{v_{0}} \frac{\alpha \Gamma K_{1}(\alpha \Gamma) J_{0}(\alpha \sin \theta)}{\Gamma^{2}+\sin ^{2} \theta} \sin \theta, \text { when } \Gamma \rightarrow 0, \text { but } \alpha \Gamma \neq 0,
$$

which is the expression already obtained by Dnestrovskii \& Kostomarov (1959b).

\section{Numerical Results and Discussion}

In Fig. 1, results concerning the radiation loss $P(\omega)$ are presented for small $\alpha$ ( $=\omega a / c_{0}$ ) and for large $\alpha$. In the region of intermediate values of $\alpha$ the low-frequency curve as well as the high-frequency curve are continued till the crossover point of these curves. The dots also shown are the results obtained by Dnestrovskii \& Kostomarov (1959a). These were obtained by numerical solution of the integral equation (see the introduction). Comparison of the values obtained by Dnestrovskii \& Kostomarov with our ones indicates reasonable agreement. We observe that for small $\alpha$ there is a slight increase of $P(\omega)$ with increasing $\alpha$. This can be explained by the fact that with an increase of $\alpha$ the aperture becomes more radiating. The maximum in the curve is arrived at $\alpha=0.6$; then, the influence of a larger decay of the incident field becomes visible and $P(\omega)$ decreases with increasing $\alpha$. We remark that the slight increase of $P(\omega)$ for small $\alpha$ does not follow from the results of Dnestrovskii \& Kostomarov (see the values at $\alpha=0.5$ ). The cause of the discrepancy has not been traced, but is very likely due to discretization errors in their numerical conversion of the integral equation, since a $5 \%$ error is sufficient to remove this increase.

In Fig. 2, results for the total radiation loss $Q$ are presented. The values $Q=$ $\int_{0}^{\infty} P(\omega) d \omega$ are obtained from a numerical integration of the values of $P(\omega)$, switching from the results for small $\alpha$ to the ones for large $\alpha$ at the crossover point of the relevant curves. From Fig. 2, we observe an almost linear behaviour

$$
Q \simeq 0.092 \frac{q^{2}}{\varepsilon_{0} a} \frac{v_{0}^{2}}{c_{0}^{2}}\left(1-\frac{v_{0}^{2}}{c_{0}^{2}}\right)^{-\frac{1}{2}} \text { when } \frac{v_{0}^{2}}{c_{0}^{2}}\left(1-\frac{v_{0}^{2}}{c_{0}^{2}}\right)^{-\frac{1}{2}}>2\left(\frac{v_{0}}{c_{0}}>0.9\right) .
$$

For lower velocities this formula can be used as an upper bound of the total radiation loss $Q$.

In case of ultrarelativistic velocity of the charge $\left(v_{0} / c_{0} \rightarrow 1\right)$, the total radiation loss becomes

$$
Q \simeq 0.092 \frac{q^{2}}{\varepsilon_{0} a}\left(1-\frac{v_{0}^{2}}{c_{0}^{2}}\right)^{-\frac{1}{2}}, \text { when } \frac{v_{0}}{c_{0}} \rightarrow 1 .
$$

We note that in the case of ultrarelativistic velocity of the charge Dnestrovskii \& Kostomarov (1959b) obtained the expression (7.2) with the factor $1 / 4 \pi \simeq 0.079$ instead of 0.092 . The discrepancy between these results is due to the fact that these authors used a high-frequency approximation for large $\alpha$ as well as for small $\alpha$. Hemp (1973) suggested, that in case of ultrarelativistic velocity, the total radiation loss is twice the energy contained in the field of the charge in free space for which $r>a$. He obtained expression (7.2) with the factor $\frac{3}{32} \simeq 0.094$ instead of 0.092. In the case of ultrarelativistic velocity, this result approximates our result very well. 


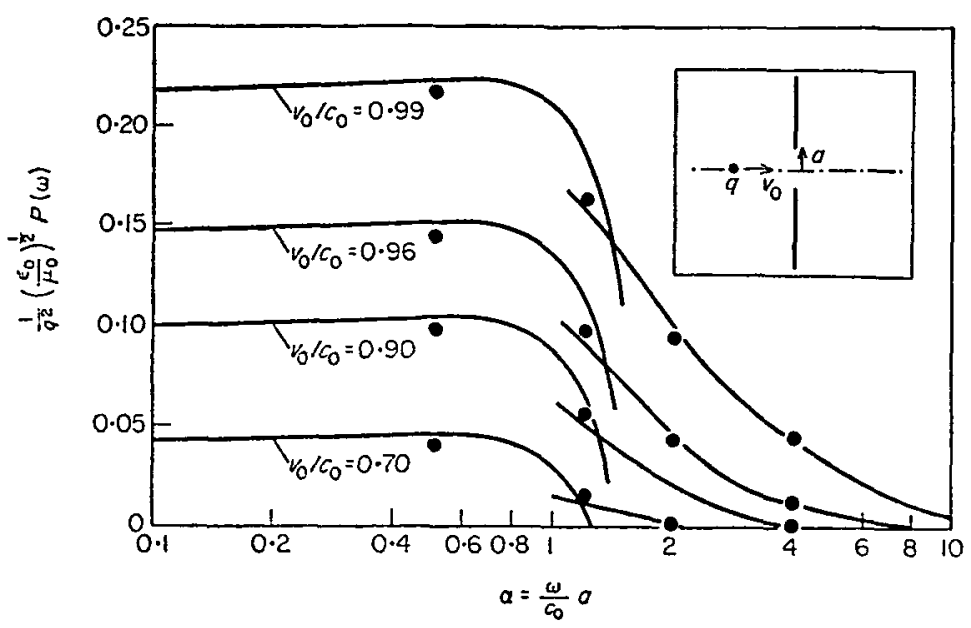

FIG. 1. Radiation loss $\left(1 / q^{2}\right)\left(\epsilon_{0} / \mu_{0}\right)^{\frac{1}{2}} P(\omega)$ as a function of $\alpha=\left(\omega / c_{0}\right) a$, with $v_{0} / c_{0}=0.99,0.96,0.90$ and 0.70 , respectively. The dots are the numerical results obtained by Dnestrovskii \& Kostomarov (1959a).

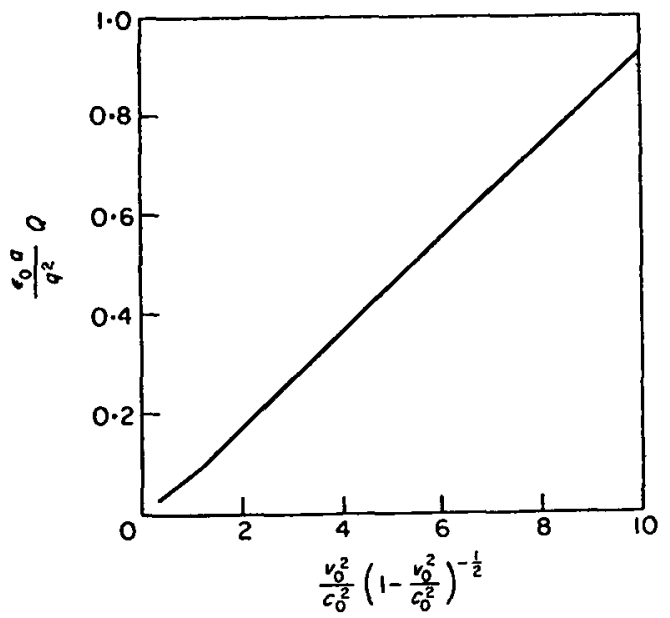

Fig. 2. Total radiation loss $\left(\epsilon_{0} a / q^{2}\right) Q$ as a function of $\left(v_{0}^{2} / c_{0}^{2}\right)\left(1-v_{0}^{2} / c_{0}^{2}\right)^{-\frac{1}{2}}$.

The author would like to thank Professor D. S. Jones for his interest concerning the research presented in this paper. This work was carried out in the Department of Mathematics, University of Dundee, Scotland, while the author was in receipt of a scholarship of the Niels Stensen Stichting, Amsterdam. This financial support is gratefully acknowledged.

\section{REFERENCES}

Bolotovskil, B. M. \& VoskresenskiI, G. V. 1966 Sov. Phys.-Usp. 9, 73-96.

BolotovskiI, B. M. \& VoskresenskiI, G. V. 1968 Sov. Phys.-Usp. 11, 143-162.

Coluins, W. D. 1961 Q. Jl Mech. appl. Math. 14, 101-117.

Dnestrovskil, Yu. N. \& Kostomarov, D. P. 1959a Sov. Phys. Dokl. 4, 132-135.

Dnestrovski, Yu. N. \& Kostomarov, D. P. $1959 b$ Sov. Phys. Dokl. 4, 158-160. 
Hazeltine, R. D., Rosenbluth, M. N. \& Sessler, A. M. 1971 J. math. Phys. 12, 502-514. HEMP, J. 1974 Q. Jl. Mech. appl. Math. 27, 255-264.

Jones, D. S. 1956 Communs Pure appl. Math. 9, 713-746.

Jones, D. S., 1965a Proc. Camb. Phil. Soc. 61, 223-245.

Jones, D. S. $1965 b$ Proc. Camb. Phil. Soc. 61, 247-270.

Newby, J. C. 1972 Proc. Camb. Phil. Soc. 71, 527-543.

OBERHETTINGER, F. \& BADI, L. 1973 Tables of Laplace transforms. Berlin: Springer-Verlag. Thomas, D. P. 1968 Q. Jl Mech. appl. Math. 21, 51-65.

Watson, G. N. 1966 A treatise on the theory of Bessel functions. Cambridge: University Press. Williams, W. E. 1962 Proc. $R$. Soc., Lond. A 267, 77-87.

Williams, W. E. 1972 Proc. Camb. Phil. Soc. 71, 423-430.

\section{Appendix}

\section{Asymptotic development of $B(v)$}

In this Appendix we derive the first terms of an asymptotic series of

with

$$
B(v)=-\frac{q}{2 \pi} \frac{d}{d v} A(v)
$$

$$
A(v)=\int_{0}^{v} w K_{0}(\alpha \Gamma w) \frac{\cosh \left\{\alpha\left(v^{2}-w^{2}\right)^{\frac{1}{2}}\right\}}{\left(v^{2}-w^{2}\right)^{\frac{1}{2}}} d w .
$$

With the substitution $w=\boldsymbol{u}^{\ddagger}$ and $v=t^{\ddagger}$, we obtain

$$
A\left(t^{\frac{1}{2}}\right)=\frac{1}{2} \int_{0}^{t} K_{0}\left(\alpha \Gamma u^{\frac{1}{2}}\right) \frac{\cosh \left\{\alpha(t-u)^{\frac{1}{2}}\right\}}{(t-u)^{\frac{1}{t}}} d u .
$$

Taking the Laplace transform of this convolution and using the Laplace transform of $K_{0}\left(\alpha \Gamma t^{t}\right)$ and $t^{-t} \cosh \left(\alpha t^{t}\right)$ (Oberhettinger \& Badii, 1973, formulae 13.33 and 9.42), we obtain

$$
\int_{01}^{\infty} A\left(t^{\frac{1}{3}}\right) \exp (-p t) d t=\frac{-\pi^{\frac{1}{2}}}{4 p^{\frac{3}{2}}}\left[\exp \left\{\frac{\alpha^{2}\left(\Gamma^{2}+1\right)}{4 p}\right\} E i\left(\frac{-\alpha^{2} \Gamma^{2}}{4 p}\right)\right], \quad \operatorname{Re}(p)>0,
$$

in which $-E i(-x)$ denotes the exponential integral

$$
\begin{aligned}
-E i(-x) & =\int_{x}^{\infty} \frac{\exp (-t)}{t} d t \\
& =-\gamma-\ln x-\sum_{n=1}^{\infty} \frac{(-x)^{n}}{n \cdot n !}, \quad x>0,
\end{aligned}
$$

where $\gamma=0.577216 \ldots$ is Euler's constant. Hence, the first few terms of a power series development of (A.4) become

$$
\begin{aligned}
& \int_{0}^{\infty} A\left(t^{\frac{1}{2}}\right) \exp (-p t) d t=\frac{\pi^{\frac{1}{2}}}{4 p^{2}}\left[1+\frac{\alpha^{2}\left(\Gamma^{2}+1\right)}{4 p}+\frac{\alpha^{4}\left(\Gamma^{2}+1\right)^{2}}{32 p^{2}}+\cdots\right] \times \\
& \times\left[-\gamma-\ln \left(\frac{\alpha^{2} \Gamma^{2}}{4 p}\right)+\frac{\alpha^{2} \Gamma^{2}}{4 p}-\frac{\alpha^{4} \Gamma^{4}}{64 p^{2}}+\cdots\right], \quad \operatorname{Re}(p)>0 .
\end{aligned}
$$

Combining the terms in (A.6), taking the inverse transform of each term with use of the relations, see also Oberhettinger \& Badii (1973),

$$
\int_{0}^{\infty} t^{n-\frac{1}{2}} \exp (-p t) d t=\frac{\left(n-\frac{1}{2}\right) !}{p^{n+\frac{1}{2}}}, \quad \operatorname{Re}(p)>0, n=0,1,2, \ldots,
$$




$$
\begin{aligned}
\int_{0}^{\infty} t^{n-\frac{1}{2}}\left\{1+\frac{1}{3}\right. & \left.+\cdots+\frac{1}{2 n-1}-\gamma-\ln \left(a t^{\frac{1}{2}}\right)\right\} \exp (-p t) d t \\
= & \frac{1}{2} \frac{\left(n-\frac{1}{2}\right) !}{p^{n+\frac{1}{2}}}\left\{-\gamma-\ln \left(\frac{a^{2}}{4 p}\right)\right\}, \quad \operatorname{Re}(p)>0, n=1,2,3, \ldots,
\end{aligned}
$$

we obtain

$A\left(t^{\frac{1}{3}}\right)=t^{\frac{1}{1}}\left\{1-\gamma-\ln \left(\alpha \Gamma t^{\frac{1}{3}}\right)\right\}+\frac{1}{12} \alpha^{2} \Gamma^{2} t^{\frac{3}{3}}+\frac{1}{6} \alpha^{2}\left(\Gamma^{2}+1\right) t^{\frac{3}{2}}\left\{\frac{4}{3}-\gamma-\ln \left(\alpha \Gamma t^{\frac{1}{3}}\right)\right\}+o\left(\alpha^{3}\right)$, (A.7) when $c_{0} / v_{0}$ is not too large, which is certainly true for the values of practical interest, say $1<c_{0} / v_{0}<2$. From (A.1) with $v=t^{\ddagger}$, we have

$$
B(v)=\frac{q}{2 \pi}\left[\gamma+\ln (\alpha \Gamma v)-\frac{1}{4} \alpha^{2} \Gamma^{2} v^{2}+\frac{1}{2} \alpha^{2}\left(\Gamma^{2}+1\right) v^{2}\{-1+\gamma+\ln (\alpha \Gamma v)\}+o\left(\alpha^{3}\right)\right] .
$$

